\title{
CCD astrometry of faint compact extragalactic radio sources. III. ${ }^{\star}$
}

\author{
E. Costa ${ }^{\star \star}$ \\ Departamento de Astronomía, Universidad de Chile, Casilla 36-D Santiago, Chile
}

Received 27 June 2001 / Accepted 2 October 2001

\begin{abstract}
Optical positions relative to the Hipparcos Catalogue (ESA 1997) have been obtained for the optical counterparts of 38 faint $(B \sim 20-24)$ southern compact extragalactic radio sources (CERS). Many of these sources are not detectable - or their coordinates not adequately measurable - by means of direct photography with a widefield telescope, so the positions were determined using a multi-step procedure involving CCD and photographic observations. This method responds both to the requirement of attaining small positional errors, and the need to refer the positions to a standard astrometric system. Estimated precisions as good as 50 mas were achieved. For 16 of the above objects we are proposing a completely new optical identification or providing a revised finder. A comparison of our optical positions with available VLBI radio positions is presented. The residuals obtained are in most cases consistent with the precision of our optical data, and provide an evaluation of these CERS as radio/optical frame link sources (or their identification as astrophysically interesting astrometric outliers). Twenty nine of the CERS observed are either defining or candidate fiducial objects of the present International Celestial Reference System (ICRS, Ma et al. 1998), so the optical data we have obtained for them is potentially useful to help mantain and possibly improve the current link of the Hipparcos reference frame to the ICRS.
\end{abstract}

Key words. astrometry - reference systems

\section{Introduction}

In this paper we give new results of a program to identify the optical counterparts, and determine precise optical positions with respect to the Hipparcos frame, of faint ( $B \sim 20-24)$ CERS, being carried out at the Estación Astronómica de Cerro El Roble (EACR), Cerro Tololo Interamerican Observatory (CTIO) and Las Campanas Observatory (LCO).

In two previous contributions (Costa \& Loyola 1999, hereafter Paper I; and Costa 2001, hereafter Paper II) we presented a detailed description of the program and the results of the exploration of the fields of 61 CERS. Positions were given for the optical counterparts of 43 CERS, and 18 new optical identifications were proposed. Here we present the results of the exploration of the fields of 42 CERS. Targets were selected from the NRL/USNO proposed reference frame list (see e.g. Johnston et al. 1995) and the list of Jauncey et al. (1989). A variety of sources were observed, among them suspected empty fields, CERS with very faint, provisional, optical identifications and CERS with optical counterparts showing evidence of structure. For 16 of these CERS we are either proposing a completely

* Figures 1-16 are only available in electronic form at http://www .edpsciences.org

** e-mail: costa@das.uchile.cl new optical identification, or providing a revised finding chart. Coordinates are given for 38 of them.

\section{Observations}

A detailed description and justification of the observational procedure is given in Sect. 2 of Paper I.

The optical positions of the CERS were obtained by means of a hybrid method involving CCD and wide-field photographic observations. The CCD observations allow for precise astrometry to faint limits in the local field of the targets, and the photographic observations permit, by means of a multi-step procedure in which ad-hoc secondary and tertiary reference frames are established, to link the former to a standard reference system. As the standard (primary) reference system we have adopted the Hipparcos Catalogue.

The photographic observations were carried out with the flat-field 70/100/210 cm Maksutov Astrograph (field: $5^{\circ} \times 5^{\circ}$, scale: $99.4^{\prime \prime} / \mathrm{mm}$ ) at the EACR. Two forming gas hypersensitized Kodak IIIaJ plates, one long exposure (50 min, typically) and one short exposure (2 min), were taken centered on each CERS. Two different exposure plates are necessary to minimize the magnitude error introduced by the fact that we are dealing with reference stars that span a large range in magnitude $(B \sim 7-18)$, as 
is the case of the primary, secondary and tertiary reference system's stars. A Schott GG385 filter was used. Although not ideal, the use of the blue bandpass was dictated by emulsion availability.

The CCD observations were secured with the $1.5 \mathrm{~m}$ telescope at CTIO and the $2.5 \mathrm{~m}$ telescope at LCO. Both telescopes provide a very similar set-up in terms of scale and field: $0.24^{\prime \prime} /$ pixel, $8.19^{\prime} \times 8.19^{\prime}$, CTIO and $0.26^{\prime \prime} /$ pixel, $8.87^{\prime} \times 8.87^{\prime}$, LCO. In both cases the CCD detectors used were Tektronix $2048 \times 2048$ chips with $24 \mu$ pixels. Five frames were typically obtained for each target, which were registered and then combined to produce a single work image. Extensive testing showed that $600 \mathrm{~s}$ exposures (CTIO; $300 \mathrm{~s}$ LCO) were necessary to obtain a reasonable signal to noise ratio for the sources, without saturating the tertiary reference stars (these latter, because of the way they are chosen, turn out to be stars roughly in the magnitude range $B \sim 15-18$ ). A Johnson $B$ filter was employed for consistency with the photographic observations. In optimum seeing conditions $\left(F W H M \leq 1.0^{\prime \prime}\right)$ - frequent both at CTIO and LCO - we estimate that the limit of detection of our survey is $B \sim 24$.

In all but 7 cases the CCD frames and the plate material were obtained at similar epochs, ensuring a negligible error contribition from the unknown proper motions of the intermediate reference stars. Table 1 gives the epochs of the observations, together with information related to the identifications of the sources. The approximate $B$ magnitudes given for the newly identified optical counterparts (and those given for sources that were found to have varied considerably in brightness) were estimated from their signal to noise ratios on the combined CCD frames. CT indicates that the CCD observations were made at CTIO; LC that they were made at LCO. An asterisk in the Remarks column indicates that additional comments are given in Sect. 5 (Notes on individual objects).

Finding charts for the newly identified optical counterparts, and for those objects which in our opinion required a revised finder (see Sect. 5, Notes on individual objects), are presented in Figs. 1 to 16. They will appear only in the on-line edition of the journal.

\section{Reductions}

Details on the reduction procedure can be found in Sect. 3 of Paper I.

The $X, Y$ coordinates of the reference stars on the photographic plates were measured with a digital Zeiss--Jena Ascorecord measuring machine. The plates were measured both in the direct and reverse positions, in an effort to cancel possible systematic errors in the $X, Y$ values. Six term quadratic relations were used in the reductions. Third order terms were not included because the flat field of the Maksutov Astrograph is almost free of distortions; and, since all plates were taken near culmination, refraction third order terms were not important. Excluding stars near the edges of the plates, close pairs and bright stars (the brightest usable Hipparcos stars have $V \sim 7$ ), on the average roughly 60 homogeneously distributed primary stars were identified in the fields of the sources. The standard deviation of the differences between calculated and catalogue values of the Hipparcos stars was $0.23^{\prime \prime}$ in $X$ and $0.21^{\prime \prime}$ in $Y$. These sigmas were calculated making no distinction between bright and faint stars.

The five CCD frames taken of each CERS were first calibrated, and then registered and combined, using standard IRAF (version 2.11.3, NOAO, University of Arizona) tasks to produce the final image from which the $X, Y$ coordinates of the PSF centroids of the CERS and the tertiary stars were extracted. This latter step was carried out by means of the PEAK task within the DAOPHOT package (Stetson 1987).

\section{Results and errors}

The resulting optical positions with respect to the Hipparcos catalogue are presented in Table 2.

Positions based on CCD observations made at CTIO are identified as CT in the Remarks column; those based on CCD observations made at LCO as LC. Since the Hipparcos catalogue was constructed to coincide with the ICRF (Kovalewsky et al. 1997), and therefore can be considered an extension to the optical domain of the extragalactic radio reference frame, our optical positions are in the system of the ICRF. The first column gives the IAU designation of the sources, the second and fourth columns their J2000.0 right ascensions and declinations, and the third and fifth columns their corresponding total internal errors.

A detailed description of the relations used to estimate the above errors is given in Sect. 5 of Paper I. The total error contribution ot the Hipparcos Catalogue varied for the present fields between 1 and 13 mas in RA, and between 1 and 12 mas in DEC. The errors of measurement (CCD images) of the tertiary stars image centroid positions varied this time between 3 and 21 mas in $X$, and between 3 and 14 mas in $Y$; those of the measurement of the source image centroid position varied between 5 and 130 mas in $X$, and between 4 and 110 mas in $Y$. These errors (sigmas) have to be interpreted with caution; it must be kept in mind that they have been derived from only five (and in some cases fewer) independent settings.

\section{Notes on individual objects}

0013-005: The large positional errors estimated for this bright object are a consequence of the poor distribution of tertiary reference stars around the optical counterpart. 0022-423: Torres \& Wroblewski (1987) claim the optical identification of this source, but the optical counterpart is not visible in their finding chart and they could not determine coordinates on account of its faintness. O'Dea et al. (1991) also observed this source based on a privately communicated identification by Peacock. They do not provide a finder nor attempt to classify the optical counterpart, but they do assign a red magnitude of 21 
Table 1. Identification of the sources.

\begin{tabular}{|c|c|c|c|c|c|c|c|}
\hline $\begin{array}{c}\text { IAU } \\
\text { Designation }\end{array}$ & $\begin{array}{l}\text { Plate } \\
\text { Epoch }\end{array}$ & $\begin{array}{c}\text { CCD } \\
\text { Epoch }\end{array}$ & & $\begin{array}{l}\text { Approx. } \\
\text { Magnitude }\end{array}$ & Nature & $\begin{array}{l}\text { Finding } \\
\text { Chart }\end{array}$ & Remarks \\
\hline $0013-005$ & 96.10 .11 & 96.07 .18 & $\mathrm{CT}$ & 19.5 & QSO & 10 & $*$ \\
\hline $0019-000$ & 90.10 .14 & 96.07 .17 & $\mathrm{CT}$ & 21.5 & Gal & 11 & \\
\hline $0022-423$ & 99.11 .04 & 96.07 .16 & $\mathrm{CT}$ & 22 & Gal? & 1 & $*$ \\
\hline $0146+056$ & 90.10 .12 & 96.07 .17 & $\mathrm{CT}$ & 21 & QSO & 6 & $*$ \\
\hline $0334-546$ & 98.10 .22 & 97.11 .25 & $\mathrm{CT}$ & 20.5 & QSO & 20 & * \\
\hline $0500+019$ & 88.11 .09 & 95.12 .21 & $\mathrm{CT}$ & 22 & QSO? & 7 & $*$ \\
\hline $0503-608$ & 98.11 .21 & 97.11 .26 & $\mathrm{CT}$ & 21 & QSO & $\mathbf{1}, 4$ & $*$ \\
\hline \multirow[t]{2}{*}{$0516-621$} & 99.01 .14 & 95.12 .19 & $\mathrm{CT}$ & 22 & QSO & 1 & $*$ \\
\hline & & 98.12 .22 & $\mathrm{LC}$ & & & & \\
\hline \multirow[t]{2}{*}{$0727-115$} & 99.03 .05 & 95.12 .19 & $\mathrm{CT}$ & 22.5 & QSO? & 1 & $*$ \\
\hline & & 98.12 .23 & $\mathrm{LC}$ & & & & \\
\hline \multirow[t]{2}{*}{$0733-174$} & 99.03 .07 & 95.12 .21 & $\mathrm{CT}$ & & $\mathrm{EF}$ & & \\
\hline & & 98.1221 & $\mathrm{LC}$ & & & & \\
\hline \multirow[t]{2}{*}{$0736-332$} & 99.03 .14 & 95.12 .22 & $\mathrm{CT}$ & 23.5 & QSO? & 1 & $*$ \\
\hline & & 98.12 .22 & $\mathrm{LC}$ & & & & \\
\hline $0738-674$ & 99.03 .06 & 95.12 .20 & $\mathrm{CT}$ & 20 & QSO & 19 & \\
\hline \multirow[t]{2}{*}{$0809-493$} & 99.03 .08 & 95.04 .23 & $\mathrm{CT}$ & 24 & QSO? & 1 & $*$ \\
\hline & & 98.12 .21 & $\mathrm{LC}$ & & & & \\
\hline $0920-397$ & 90.01 .21 & 98.12 .21 & $\mathrm{LC}$ & 19 & QSO & 15 & \\
\hline $1145-676$ & 94.05 .03 & 96.07 .18 & $\mathrm{CT}$ & 20.5 & QSO? & 9 & $*$ \\
\hline $1245-197$ & 99.05 .18 & 95.04 .22 & $\mathrm{CT}$ & 21 & QSO & $\mathbf{1}, 18$ & * \\
\hline \multirow[t]{2}{*}{$1308-220$} & 90.03 .24 & 96.07 .17 & $\mathrm{CT}$ & 21.5 & QSO? & 1 & $*$ \\
\hline & & 97.02 .08 & $\mathrm{CT}$ & & & & \\
\hline \multirow[t]{2}{*}{$1309-220$} & 90.03 .24 & 96.07 .17 & $\mathrm{CT}$ & 20 & Gal & $\mathbf{1}, 3$ & $*$ \\
\hline & & 97.02 .08 & $\mathrm{CT}$ & & & & \\
\hline $1320-446$ & 97.05 .06 & 96.07 .18 & $\mathrm{CT}$ & 20 & QSO? & 17 & \\
\hline $1351-018$ & 99.05 .19 & 95.04 .21 & $\mathrm{CT}$ & 21 & QSO & 20 & $*$ \\
\hline $1352-104$ & 99.05 .14 & 95.04 .23 & $\mathrm{CT}$ & 18 & QSO? & 12 & \\
\hline $1406-076$ & 96.05 .13 & 96.07 .16 & $\mathrm{CT}$ & 20 & QSO & 5 & * \\
\hline $1421-490$ & 99.05 .19 & 95.04 .24 & $\mathrm{CT}$ & & $\mathrm{EF} ?$ & & $*$ \\
\hline $1430-178$ & 98.06 .30 & 96.07 .17 & $\mathrm{CT}$ & 19.5 & QSO & 20 & \\
\hline \multirow[t]{2}{*}{$1540-828$} & 99.05 .13 & 95.04 .22 & $\mathrm{CT}$ & 22 & QSO? & 1 & * \\
\hline & & 95.04 .23 & $\mathrm{CT}$ & & & & \\
\hline $1622-253$ & 99.08 .03 & 95.05 .04 & $\mathrm{LC}$ & 22 & QSO? & 1 & * \\
\hline $1622-297$ & 98.06 .30 & 96.07 .16 & $\mathrm{CT}$ & 20.5 & QSO & 17 & \\
\hline $1732-598$ & 93.06 .18 & 96.07 .16 & $\mathrm{CT}$ & 21 & QSO? & 9 & \\
\hline $1758-651$ & 93.06 .15 & 96.07 .17 & $\mathrm{CT}$ & 18 & QSO? & 20 & $*$ \\
\hline $1903-802$ & 98.09 .24 & 96.07 .18 & $\mathrm{CT}$ & 19 & QSO & 2 & $*$ \\
\hline $1929-457$ & 93.07 .20 & 96.07 .17 & $\mathrm{CT}$ & 20.5 & QSO & $\mathbf{1}, 16$ & * \\
\hline $1936-155$ & 98.09 .21 & 96.07 .19 & $\mathrm{CT}$ & 19 & QSO & 8 & \\
\hline $1947+079$ & 91.08 .09 & 95.05 .06 & $\mathrm{LC}$ & 21 & QSO & 20 & \\
\hline $2025-538$ & 93.10 .07 & 96.07 .16 & $\mathrm{CT}$ & 21 & QSO? & 9 & \\
\hline $2044-027$ & 95.08 .25 & 96.07 .17 & $\mathrm{CT}$ & 20 & QSO & 14 & $*$ \\
\hline $2056-369$ & 95.09 .24 & 96.07 .19 & $\mathrm{CT}$ & 23 & QSO? & 6 & $*$ \\
\hline $2059-786$ & 95.08 .27 & 96.07 .16 & $\mathrm{CT}$ & 22 & QSO? & 1 & $*$ \\
\hline $2105-489$ & 96.10 .09 & 96.07 .18 & $\mathrm{CT}$ & 20 & QSO? & 9 & \\
\hline $2109-811$ & 92.09 .23 & 96.07 .16 & $\mathrm{CT}$ & 21 & QSO? & $\mathbf{1}, 9$ & $*$ \\
\hline $2252-089$ & 96.09 .03 & 96.07 .18 & $\mathrm{CT}$ & 23 & QSO & $\mathbf{1}, 6$ & $*$ \\
\hline $2259-375$ & 96.09 .05 & 96.07 .16 & $\mathrm{CT}$ & & $\mathrm{EF} ?$ & & $*$ \\
\hline $2337-334$ & 96.10 .11 & 96.07 .17 & $\mathrm{CT}$ & 22 & QSO? & $\mathbf{1}, 6$ & $*$ \\
\hline
\end{tabular}

1 Indicates a newly identified optical counterpart or that a revised finder is being provided; LC stands for Las Campanas;

CT stands for Cerro Tololo. 


\section{References to finding charts}

[1] This paper

[2] Anguita, C., Campusano, L. E., Torres, C., et al. 1979, AJ, 84, 718

[3] Bolton, J. G., \& Ekers, J. 1966, AJP, 19, 275

[4] Bolton, J. G., \& Savage, A. 1977, AJP Ap. Supp., 41, 25

[5] Bolton, J. G., Shimmins, A. J., Wall, J. V., et al. 1975, AJP Ap. Supp., 34, 1

[6] Drinkwater, M. J., Webster, R. L., Francis, P. J., et al. 1997, MNRAS, 284, 85

[7] Fugmann, W., Meisenheimer, K., \& Roser, H. J. 1988, A\&AS, 75, 173

[8] Jauncey, D. L., Batty, M. J., Gulkis, S., et al. 1982, AJ, 87, 763

[9] Jauncey, D. L., Savage, A., Morabito, D. D., et al. 1989, AJ, 98, 54

[10] Johnson, K.H. 1974, AJ, 79, 1006

[11] McEwan, N. J., Browne, I. W. A., \& Crowther, J. H. 1975, Mem. RAS, 80, 1

[12] Peterson, B. A., Bolton, J. G., \& Shimmins, A. J. 1973, Ap. Lett., 15, 109

[13] Savage, A., Bolton, J. G., \& Trett, J. 1982, AJP, 35, 207

[14] Smith, H. E., \& Spinrad, H. 1980, ApJ, 236, 419

[15] Spinrad, H., \& Kron, R. G. 1979, ApJS, 41, 701

[16] Shimmins, A. J., Bolton, J. G., Peterson, B. A., et al. 1971, Ap. Lett., 8, 139

[17] Torres, C., \& Wroblewski, H. 1984, A\&A, 141, 271

[18] Walter, H. G., \& West, R. M. 1980, A\&A, 86, 1

[19] White, G. L., Batty, M. J., Bunton, J. D., et al. 1987, MNRAS, 227, 705

[20] Zacharias, N., Zacharias, M. I., Hall, D. M., et al. 1999, AJ, 118, 2511

to it. Figure 1 shows the identification we are proposing, which seems to coincide with the position given by Torres and Wroblewski. Our CCD images show some evidence of structure, indicating that it could be a galaxy.

0146+056: This source was first optically identified by Radivich \& Kraus (1971). Drinkwater et al. (1997, hereafter Drinkwater) provide however a better finder. The optical counterpart seems to have been much brighter when observed by Radivich. Variable? The high radio-optical residual we obtain in $\mathrm{RA}$ is consistent with that given by Drinkwater.

0334-546: Variable?

$\mathbf{0 5 0 0 + 0 1 9}$ : In spite of the poor seeing conditions in which our CCD material was obtained, our images show evidence of structure. See extensive note by Fugmann et al. (1988), who barely resoved the optical counterpart. For this source we obtain very high radio-optical RA and DEC residuals, which could probably be attributed to our mediocre observational material. Drinkwater also obtains large residuals for this object, but they are completely inconsistent with ours.

0503-608: Three optical objects are seen in the vicinity of the radio source location. Bolton \& Savage (1977) claim the optical identification of this source but their finding chart does not resolve them. In Fig. 2 we indicate the optical object which has the smallest radio-optical residuals. 0516-621: No optical counterpart was known for this source. Figure 3 shows the identification we are proposing. 0727-115: Hughes et al. (1992) observed this source, but did not provide an identification. Figure 4 shows the identification we are proposing.

0736-332: No optical counterpart was known for this source. Figure 5 shows the identification we are proposing. Very faint. Visible only in the LCO $2.5 \mathrm{~m}$ telescope frames; not detected with the CTIO $1.5 \mathrm{~m}$ telescope. A very faint and diffuse (not measurable) object is seen to the north, very close to that which we are indicating as the optical counterpart. This latter object cannot be ruled out as the true counterpart.

0809-493: No optical counterpart was known for this source. Figure 6 shows the identification we are proposing.

1145-676: Optically variable. This object was roughly two magnitudes fainter when observed by Jauncey et al. (1989).

1245-197: Originally identified by Walter \& West (1980). Here we provide an improved finding chart (Fig. 7).

1308-220 (3C 283) and 1309-220 (PKS 1309-220): There is considerable confusion in the literature about the identification of these sources. PKS 1309-220 is included in the Parkes Catalogue (see e.g. Bolton et al. 1979), and its optical counterpart has been identified as a faint galaxy by Bolton \& Ekers (1966). This identification was confirmed by Costa \& Loyola (1994), but they incorrectly named the source as 3C 283. 3C 283 has been observed in the radio domain by Ulvestad et al. (1981), but they incorrectly indicate as its optical counterpart the above galaxy. Torres \& Wroblewski (1997) correctly identified the field of $3 \mathrm{C} 283$, but they could not detect the optical counterpart. Here we provide an improved finder for PKS 1309-220 (Fig. 8) and in Fig. 9 show the optical identification we are proposing for 3C 283 .

1351-018: The high radio-optical residual we obtain in DEC is consistent with that obtained by Drinkwater; inconsistent however with that obtained by Zacharias et al. (1999, hereafter Zacharias). It should be noted that our CCD material was obtained in mediocre seeing conditions.

1406-076: Differences detected in the magnitude assigned to this object suggest that it is grossly variable. Our radio-optical residuals (small in RA; fairly large in DEC) are not consistent with those obtained by Drinkwater \& Zacharias. 
Table 2. Optical positions in the ICRS system of 38 compact extragalactic radio sources.

\begin{tabular}{|c|c|c|c|c|c|}
\hline$(1)$ & $(2)$ & $(3)$ & $(4)$ & $(5)$ & $(6)$ \\
\hline IAU & RA(J2000.0) & $\epsilon_{\alpha} \cos \delta$ & DEC (J2000.0) & $\epsilon_{\delta}$ & Remark \\
\hline Designation & $\mathrm{h} \mathrm{m} \quad \mathrm{s}$ & $\pm^{\prime \prime}$ & " & $\pm^{\prime \prime}$ & \\
\hline $0013-005$ & 001611.094 & 0.10 & -001512.48 & 0.11 & $\mathrm{CT}$ \\
\hline $0019-000$ & 002225.427 & 0.09 & +001456.21 & 0.09 & $\mathrm{CT}$ \\
\hline $0022-423$ & 002442.999 & 0.08 & -420203.52 & 0.09 & $\mathrm{CT}$ \\
\hline $0146+056$ & 014922.330 & 0.10 & +055553.33 & 0.09 & $\mathrm{CT}$ \\
\hline $0334-546$ & 033553.937 & 0.07 & -543025.09 & 0.06 & $\mathrm{CT}$ \\
\hline $0500+019$ & 050321.156 & 0.09 & +020303.16 & 0.09 & $\mathrm{CT}$ \\
\hline $0503-608$ & 050401.750 & 0.08 & -604952.45 & 0.07 & $\mathrm{CT}$ \\
\hline \multirow[t]{2}{*}{$0516-621$} & 051644.936 & 0.07 & -620705.16 & 0.08 & $\mathrm{CT}$ \\
\hline & 44.923 & 0.07 & 05.35 & 0.06 & $\mathrm{LC}$ \\
\hline \multirow[t]{2}{*}{$0727-115$} & 073019.102 & 0.08 & -114112.30 & 0.08 & $\mathrm{CT}$ \\
\hline & 19.112 & 0.09 & 12.49 & 0.09 & $\mathrm{LC}$ \\
\hline $0736-332$ & 073816.937 & 0.09 & -332212.80 & 0.08 & $\mathrm{LC}$ \\
\hline $0738-674$ & 073856.472 & 0.06 & -673550.67 & 0.05 & $\mathrm{CT}$ \\
\hline \multirow[t]{2}{*}{$0809-493$} & 081108.791 & 0.11 & -492943.52 & 0.11 & $\mathrm{CT}$ \\
\hline & 08.804 & 0.10 & 43.58 & 0.10 & $\mathrm{LC}$ \\
\hline 0920-397 & 092246.421 & 0.07 & -395934.96 & 0.07 & $\mathrm{LC}$ \\
\hline $1145-676$ & 114733.373 & 0.06 & -675342.07 & 0.06 & $\mathrm{CT}$ \\
\hline $1245-197$ & 124823.888 & 0.08 & -195918.66 & 0.08 & $\mathrm{CT}$ \\
\hline $1308-220$ & 131139.355 & 0.09 & $-22 \quad 1641.31$ & 0.08 & $\mathrm{CT}$ \\
\hline $1309-220$ & 131140.105 & 0.08 & $-22 \quad 1704.44$ & 0.08 & $\mathrm{CT}$ \\
\hline $1320-446$ & 132304.254 & 0.07 & -445233.90 & 0.07 & $\mathrm{CT}$ \\
\hline $1351-018$ & 135406.888 & 0.07 & -020603.98 & 0.09 & $\mathrm{CT}$ \\
\hline $1352-104$ & 135446.488 & 0.05 & -102603.21 & 0.06 & $\mathrm{CT}$ \\
\hline $1406-076$ & 140856.487 & 0.08 & -075227.16 & 0.09 & $\mathrm{CT}$ \\
\hline $1430-178$ & 143257.709 & 0.08 & -180135.20 & 0.07 & $\mathrm{CT}$ \\
\hline $1540-828$ & 155059.022 & 0.09 & -825807.06 & 0.09 & $\mathrm{CT}$ \\
\hline $1622-253$ & 162546.904 & 0.09 & -252737.87 & 0.09 & $\mathrm{LC}$ \\
\hline $1622-297$ & 162606.010 & 0.08 & -295127.03 & 0.08 & $\mathrm{CT}$ \\
\hline $1732-598$ & 173630.862 & 0.07 & -595158.34 & 0.06 & $\mathrm{CT}$ \\
\hline $1758-651$ & 180323.512 & 0.05 & -650736.32 & 0.07 & $\mathrm{CT}$ \\
\hline $1903-802$ & 191240.082 & 0.10 & -801004.68 & 0.09 & $\mathrm{CT}$ \\
\hline $1929-457$ & 193244.890 & 0.07 & -453637.95 & 0.06 & $\mathrm{CT}$ \\
\hline $1936-155$ & 193926.655 & 0.06 & -152542.93 & 0.06 & $\mathrm{CT}$ \\
\hline $1947+079$ & 195005.545 & 0.08 & +080714.20 & 0.08 & $\mathrm{LC}$ \\
\hline $2025-538$ & 202935.041 & 0.06 & -533907.56 & 0.07 & $\mathrm{CT}$ \\
\hline $2044-027$ & 204710.355 & 0.07 & -023622.21 & 0.06 & $\mathrm{CT}$ \\
\hline $2059-786$ & 210544.926 & 0.08 & -782534.55 & 0.07 & $\mathrm{CT}$ \\
\hline $2105-489$ & 210850.800 & 0.07 & -484621.90 & 0.07 & $\mathrm{CT}$ \\
\hline $2109-811$ & 211630.869 & 0.07 & -805354.87 & 0.07 & $\mathrm{CT}$ \\
\hline $2252-089$ & 225504.192 & 0.08 & -084404.17 & 0.09 & $\mathrm{CT}$ \\
\hline $2337-334$ & 233954.515 & 0.08 & -331017.02 & 0.08 & $\mathrm{CT}$ \\
\hline
\end{tabular}

CT stands for Cerro Tololo; LC stands for Las Campanas.

1421-490: Empty field? A very faint, not measurable object is seen near the radio source position.

1540-828: No optical counterpart was known for this source. Figure 10 shows the identification we are proposing.

1622-253: No optical counterpart was known for this source. Figure 11 shows the identification we are proposing.

1758-651: Triple optical image. There is evidence of structure connecting the optical counterpart to a bright pair of objects to the north. This could explain the fairly high radio-optical DEC residual obtained, which is consistent with that obtained by Zacharias.

1903-802: The high radio-optical DEC residual we obtain is not consistent with that obtained by Zacharias. We find no evidence of an alternative, fainter, optical counterpart down to a magnitude limit of $B \sim 23$.

1929-457: Double image, just barely resolved as two objects of similar brightness in our CCD frames. Shimmins et al. (1971) claim the optical identification of this source, 
but in their finding chart the object is not resolved. Jauncey et al. (1989) also observed this source, but although they did recognize its structure, they could not resolve it. Here we give the correct identification (Fig. 12). 2044-027: Smith \& Spinrad (1980) present a discussion on the true nature of this object and provide a superb finder.

2056-369: Drinkwater claims the optical identification of this source, but in their finding chart the optical counterpart is not visible. In our CCD material the proposed counterpart is not detected; this probably on account of the fact that it was obtained in mediocre seeing conditions. 2059-786: No optical counterpart was known for this source. Figure 13 shows the identification we are proposing.

2109-811: Triple optical image partially resolved in our frames. Jauncey et al. (1989) claim the optical identification of this source, but in their finding chart the optical image is resolved in only two objects. Figure 14 shows the optical identification we are proposing. Contrary to what this figure seems to indicate, there is evidence of structure connecting the three objects. This could explain the fairly large radio-optical DEC residual.

2252-089: Drinkwater claims the optical identification of this source, but in their finding chart the optical counterpart is not visible. In Fig. 15 we present an improved finder.

2259-375: Empty field? The radio source position coincides with a diffuse, not measurable, optical structure at the limit of detection.

2337-334: Drinkwater claims the optical identification of this source, but in their finding chart the optical counterpart is not visible. In Fig. 16 we present an improved finder.

\section{Discussion}

The present results confirm that combining CCD and photographic observations it is possible to obtain "absolute" (i.e. refered to a standard astrometric system) optical positions of faint $(B \sim 20-24)$ CERS with an estimated precision as good as 50 mas. Although the ultimate precision attainable with our method is hampered by the accuracy with which the positions of the reference stars can be determined measuring the plates with a conventional manual measuring device, an important reduction of the total internal error results from the significant decrease of the error in the determination of the centroid of the optical counterpart. This error completely dominates the total internal error in a purely photographic approach, even in the case of moderately faint $(B \sim 19.5)$ objects (see Costa \& Loyola 1992). Furthermore, the method proposed is capable of precise astrometry of objects that are not measurable - or simply not visible - on a wide-field telescope plate, and very effective at exploring suspected empty fields in search faint optical counterparts.

In Table 3 we present a comparison in the ICRS with the VLBI radio position given by Ma et al. (1998), in the
Table 3. Comparison in the ICRS with the VLBI radio positions given by Ma et al. (1998). See Sect. 6 for details.

\begin{tabular}{|c|c|c|c|}
\hline \multirow{2}{*}{$\begin{array}{c}\text { IAU } \\
\text { Designation }\end{array}$} & \multicolumn{2}{|c|}{ (Radio-C) } & \multirow[t]{2}{*}{ Remarks } \\
\hline & $\Delta_{\prime \prime}^{\alpha} \delta$ & $\underset{\prime \prime}{\Delta \delta}$ & \\
\hline $0013-005$ & -0.08 & 0.03 & $\mathrm{CT}$ \\
\hline 0019-000 & 0.04 & -0.08 & $\mathrm{CT}$ \\
\hline $0022-423$ & -0.10 & -0.43 & $\mathrm{CT}$ \\
\hline $0146+056$ & 0.61 & 0.24 & $\mathrm{CT}$ \\
\hline $0334-546$ & -0.11 & -0.02 & $\mathrm{CT}$ \\
\hline $0500+019$ & 0.62 & 1.52 & $\mathrm{CT}$ \\
\hline $0503-608$ & -0.36 & -0.09 & $\mathrm{CT}$ \\
\hline \multirow{2}{*}{$0516-621$} & -0.07 & -0.23 & $\mathrm{CT}$ \\
\hline & 0.02 & -0.04 & $\mathrm{LC}$ \\
\hline \multirow[t]{2}{*}{$0727-115$} & 0.15 & -0.30 & $\mathrm{CT}$ \\
\hline & 0.01 & -0.11 & $\mathrm{LC}$ \\
\hline $0736-332$ & 0.15 & 0.02 & $\mathrm{LC}$ \\
\hline $0738-674$ & 0.14 & -0.16 & $\mathrm{CT}$ \\
\hline \multirow[t]{2}{*}{$0809-493$} & 0.11 & 0.01 & $\mathrm{CT}$ \\
\hline & -0.01 & 0.07 & $\mathrm{LC}$ \\
\hline $0920-397$ & -0.03 & -0.11 & $\mathrm{LC}$ \\
\hline $1245-197$ & 0.17 & 0.00 & $\mathrm{CT}$ \\
\hline $1320-446$ & -0.09 & 0.05 & $\mathrm{CT}$ \\
\hline $1351-018$ & 0.11 & 0.79 & $\mathrm{CT}$ \\
\hline $1352-104$ & -0.12 & -0.05 & $\mathrm{CT}$ \\
\hline $1406-076$ & -0.09 & 0.49 & $\mathrm{CT}$ \\
\hline $1430-178$ & -0.26 & -0.05 & $\mathrm{CT}$ \\
\hline $1540-828$ & 0.23 & 0.22 & $\mathrm{CT}$ \\
\hline $1622-253$ & -0.17 & -0.46 & $\mathrm{LC}$ \\
\hline $1622-297$ & 0.14 & 0.06 & $\mathrm{CT}$ \\
\hline $1758-651$ & -0.10 & -0.44 & $\mathrm{CT}$ \\
\hline $1903-802$ & -0.16 & -1.27 & $\mathrm{CT}$ \\
\hline $1936-155$ & 0.04 & -0.13 & $\mathrm{CT}$ \\
\hline $1947+079$ & -0.08 & -0.22 & $\mathrm{LC}$ \\
\hline $2059-786$ & 0.11 & -0.00 & $\mathrm{CT}$ \\
\hline $2109-811$ & -0.05 & -0.35 & $\mathrm{CT}$ \\
\hline $2252-089$ & +0.71 & 0.15 & $\mathrm{CT}$ \\
\hline Mean residual & +0.01 & -0.04 & \\
\hline Sigma & 0.18 & 0.26 & \\
\hline Standard error & 0.03 & 0.05 & \\
\hline
\end{tabular}

CT and LC stand for Cerro Tololo and Las Campanas respectively.

sense radio minus this work $(\mathrm{C})$. To determine the statistics given at the bottom of the table, observations of a same source carried out both at CTIO and LCO were taken as independent entries. Also, sources $0500+019$, 1903-802, and 2252-089 were not considered in the calculations, because their radio- $\mathrm{C}$ residuals (either in $\mathrm{RA}$ or DEC) are larger than three times the standard deviation of the mean residual of the complete sample.

Since our optical positions are displayed in essentially the same reference system as the radio positions, the individual radio- $\mathrm{C}$ differences given in Table 3 provide an evaluation of the CERS observed as possible radio/optical frame link sources; in 7 cases (namely 0516-621, 0736-332, 0809-493, 1308-220, 1540-828, 1622-253, and 2059-786) the first one available. With a 

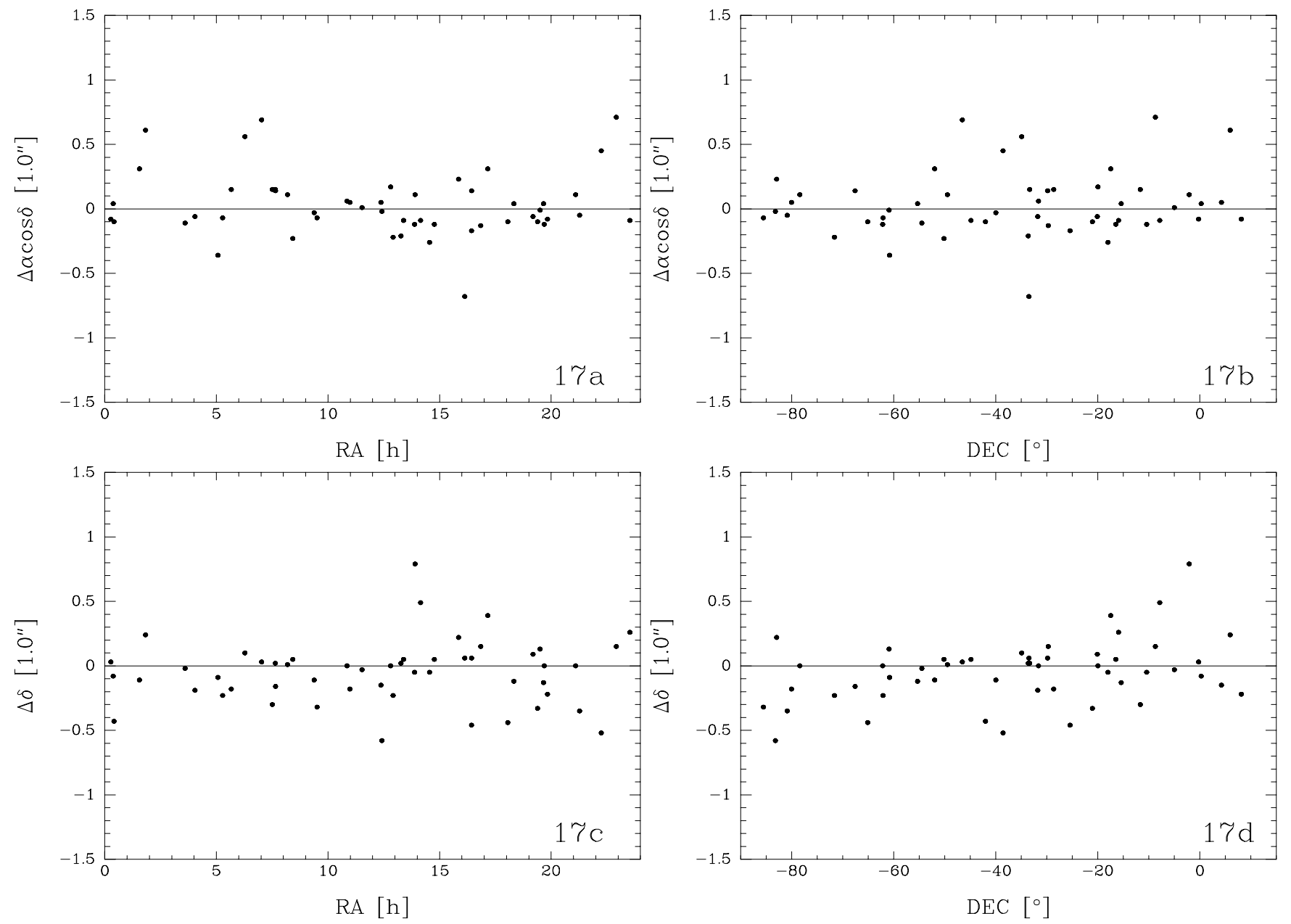

Fig. 17. a-d): (Radio - C) residuals of the comparison between our optical positions $(\mathrm{C})$ and the VLBI radio positions given by Ma et al. (1998), plotted as a function of the coordinates. The plot includes the present results and those given in Papers I and II. See Sect. 6 for details.

few exceptions (see Sect. 5, Notes on individual objects), the residuals obtained are consistent with the overall, estimated precision of our data. Although most of the objects which show high residuals are probably real astrometric outliers (i.e. the residuals are showing real offsets between their radio and optical emission centroids, in which event these CERS are potentially interesting from an astrophysical point of view), we cannot rule out the possibility that the proposed optical counterpart (either by us, or previous) may be incorrect. Also, it must be kept in mind that the errors in the measurement of the primary, secondary and tertiary reference stars used to calculate the total internal errors of our positions were average values based on previous experience (see Sect. 5.1 of Paper I), making it probable that for some objects their positional errors may have been underestimated. Furthermore, we cannot rule out the existence of an undetected magnitude equation.

Combining the results presented in Table 3 with analogous results published in Papers I and II, a comparison subset with the VLBI positions given by Ma et al. (1998) of 54 objects is obtained. After the omission of 2 sources which have (radio-C) residuals more than 3 times the standard deviation of the mean residual of the subset, and, again, considering observations of a same source carried out both at CTIO and LCO as independent entries, the following statistics is obtained:

$$
\begin{array}{ll}
\overline{\Delta \alpha \cos \delta}=+0.03^{\prime \prime} & \overline{\Delta \delta}=-0.05^{\prime \prime} \\
\sigma(\overline{\Delta \alpha \cos \delta})=0.24^{\prime \prime} & \sigma(\overline{\Delta \delta})=0.24^{\prime \prime} \\
\epsilon(\overline{\Delta \alpha \cos \delta})=0.03^{\prime \prime} & \epsilon(\overline{\Delta \delta})=0.03^{\prime \prime} .
\end{array}
$$

The $\Delta \alpha \cos \delta$ and $\Delta \delta$ radio- $\mathrm{C}$ residuals are plotted as a function of the coordinates in Figs. 17a-d. Quick inspection of these plots shows that, with the possible exception of the relation $\Delta \delta$ vs. $\delta$, there is no evidence of systematic effects.

Considering that 47 of the CERS for which a position has been determined in the course of our program are either defining or candidate fiducial radio sources of the present realization of the ICRS, the results of our survey are potentially useful to help mantain, and possibly improve, the current link of the Hipparcos reference frame to the ICRS. Although at this moment a link based on 
CERS that display optical emission is less precise than other methods that contributed to the realization of the present link (see e.g. Kovalewsky et al. 1997), it has great potential because of the large number of well-distributed link sources involved (see e.g. Zacharias et al. 1995, 1999). Within this same context, the identification of of new radio/optical frame link objects is of particular importance (see e.g. Walter \& Sovers 2000).

Acknowledgements. I am specially indebted to Prof. P. Loyola for his contributions to this and previous related programs throughout the years and to M. Wishnjewski and L. E. González for measuring the plates. I am also indebted to Cerro Tololo Interamerican Observatory for donating the plate material that made this survey possible. This work was partially financed by the Fondo Nacional de Investigación Científica y Tecnológica (proyecto No. 1970767 Fondecyt).

\section{References}

Bolton, J. G., Savage, A., \& Wright, E. 1979, AJP Ap. Supp., 46,1

Costa, E., \& Loyola, P. 1992, A\&AS, 96, 183

Costa, E., \& Loyola, P. 1994, A\&AS, 104, 503
Costa, E., \& Loyola, P. 1999, A\&AS, 139, 297

Costa, E., \& Loyola, P. 2001, A\&A, 367, 719

ESA, 1997, The Hipparcos and Tycho Catalogues, ESA SP1200, European Space Agency, vols. 1-17

Hughes, P. A., Aller, H. D., \& Aller, M. F. 1992, ApJ, 396, 469 Jauncey, D. L., Savage, A., Morabito, D. D., et al. 1989, AJ, 98,54

Johnston, K. J., Fey, A. L., Zacharias, N., et al. 1995, AJ, 110, 880

Kovalewsky, J., Lindegren, L., Perryman, M. A. C., et al. 1997, A\&A, 323, 620

Ma, C., Arias, E. F., Eubanks, T. M., et al. 1998, AJ, 116, 516

O'Dea, C. P., Baum, S. A., \& Stanghellini, C. 1991, ApJ, 380, 66

Radivich, M. M., \& Kraus, J. D. 1971, AJ, 76, 683

Stetson, P. B. 1987, PASP, 99, 191

Torres, C., \& Wroblewski, H. 1987, A\&AS, 69, 23

Walter, H. G., \& Sovers, O. J. 2000, in Astrometry of Fundamental Catalogues. The evolution from Optical to Radio Reference Frames, Astronomy \& Astrophysics Library, ISSN 0941-7834, 187

Zacharias, N., de Vegt, C., Winter, L., et al. 1995, AJ, 110, 3093

Zacharias, N., Zacharias, M. I., Hall, D. M., et al. 1999, AJ, 118,2511 